\title{
FACTORS ASSOCIATED WITH DIARRHEA PREVENTION IN ELEMENTARY SCHOOL STUDENTS IN NGAWI, EAST JAVA
}

\author{
Dian Surya Kartika'), Setyo Sri Rahardjo²), Bhisma Murti1) \\ 1)Masters Program in Public Health, Universitas Sebelas Maret \\ 2)Faculty of Medicine, Universitas Sebelas Maret
}

\begin{abstract}
Background: Good sanitation and personal hygiene are necessary condition for diarrhea prevention. This study aimed to determine factors associated with diarrhea prevention in elementary school students in Ngawi, East Java.

Subjects and Method: A cross sectional study was conducted in 25 primary schools in Ngawi, East Java, in October 2018. A sample of 225 students was selected by random sampling. The dependent variable was diarrhea prevention. The independent variables were knowledge, attitude, maternal education, hygiene facility, school sanitation, and school health unit. The data were collected by questionnaire and analyzed by a multiple logistic regression.

Results: Diarrhea prevention improved with good hygiene facility $(b=3.25 ; 95 \% \mathrm{CI}=1.61$ to 6.87; $\mathrm{p}=0.001)$, good school sanitation $(\mathrm{b}=2.24 ; 95 \% \mathrm{CI}=1.10$ to $4.7 ; \mathrm{p}=0.025)$, good canteen hygiene $(\mathrm{b}=2.99 ; 95 \% \mathrm{CI}=0.23$ to $3.44 ; \mathrm{p}=0.003)$, good school health unit facility $(b=2.60 ; 95 \% C I=1.30$ to $6.53 ; p=0.009)$, good student knowledge $(b=3.87 ; 95 \% \mathrm{CI}=2.04$ to 8.92; $\mathrm{p}<0.001)$, good attitude toward diarrhea prevention $(\mathrm{b}=2.79 ; 95 \% \mathrm{CI}=1.39$ to 6.67 ; $\mathrm{p}=0.005)$, and maternal education $\geq$ senior high school $(\mathrm{b}=4.05 ; 95 \% \mathrm{CI}=2.30$ to 11.00 ; $\mathrm{p}<0.001)$.

Conclusion: Diarrhea prevention improves with good hygiene facility, good school sanitation, good canteen hygiene, good school health unit facility, good student knowledge, good attitude toward diarrhea prevention, and maternal education $\geq$ senior high school
\end{abstract}

Keywords: diarrhea prevention, school sanitation, canteen hygiene

\section{Correspondence:}

Dian Surya Kartika. Masters Program in Public Health, Universitas Sebelas Maret. Jl. Ir. Sutami 36 A, Surakarta 57126, Central Java, Indonesia.

Email: kartikadiansurya@gmail.com. Mobile: +6282230003592

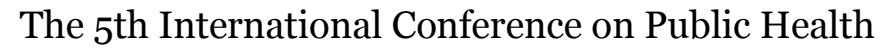

Best Western Premier Hotel, Solo, Indonesia, February 13-14, 2019 | 166

https://doi.org/10.26911/theicph.2019.02.11 